\title{
Study the Optical and Spectral Properties of the Acridine Dye As an Effective Medium in Dye Lasers
}

\author{
Afrah M. AL Hussainey $^{a} \quad$ Ruaa Kahtan Mahmoud $^{\mathrm{b}}$ Talib M. Abbas ALShafie $^{\mathrm{c}}$ \\ ${ }^{a, b}$ Department of Physics, college of Science,Babylon University, Babylon, Iraq \\ ${ }^{c}$ Department of Physics, Education for pure sciences, Babylon University,Babylon, Iraq \\ afrahalsherefy@yahoo.com_Dr.Talib_mohsen@yahoo.com
}

Submission date:- 29/5/2018 Acceptance date:- 12/6/2018 Publication date:- 29/11/2018

Keywords: Acridine, Absorption, Fluorescence, Laser Dye.

\begin{abstract}
In this study, the absorption and fluorescence spectra of the dissolved Acridine solution were studied in ethanol and different concentrations. The optical properties (absorption coefficient, linear refraction) and spectral characteristics (Fluorescence time and quantitative fluoridation efficiency) were calculated. It is observed that increasing the concentration increases the absorbance values and decreases the permeability values, thus increasing the values of the absorption coefficient and the refractive index. Also, the values of the fluorine intensity increase. The peak of the emission spectrum is transferred to the longer wavelengths.
\end{abstract}

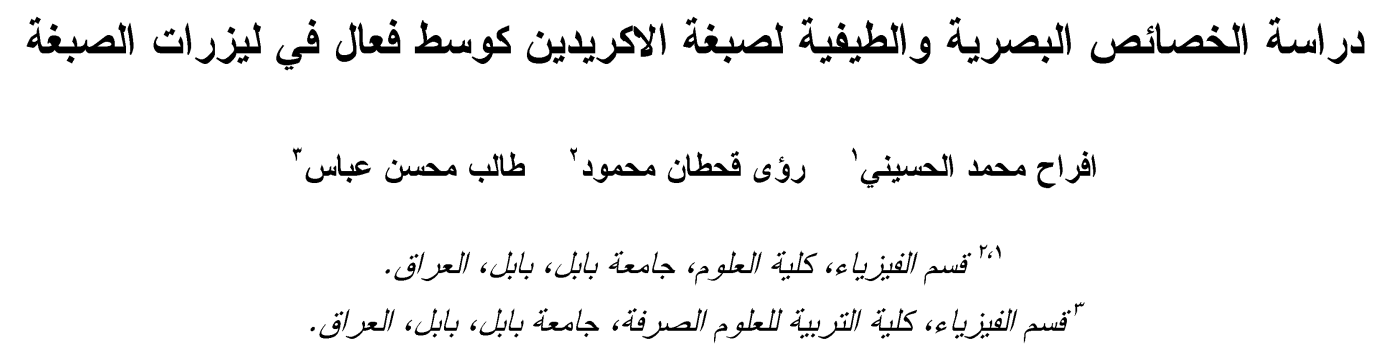

afrahalsherefy@yahoo.com_Dr.Talib_mohsen@yahoo.com 


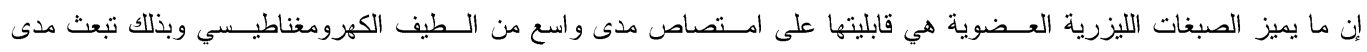

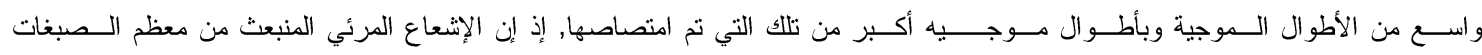

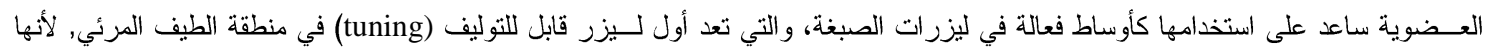

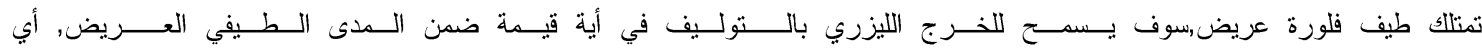

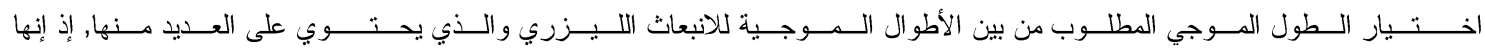

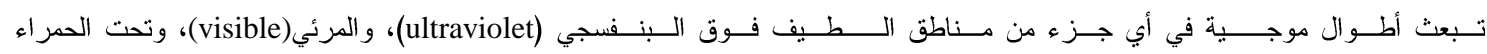

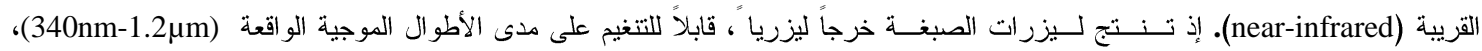

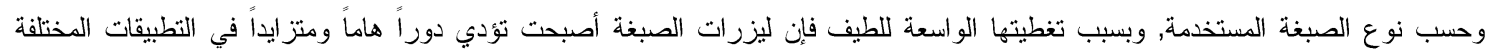
وتتثمل (در اسة الاطياف و الكيمياء الفيزيائية )[3],[2].

The Theoretical Part الجزء النظري

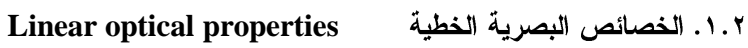
إن التفاعل الحاصل بين طبيعة وتوزيع الثحنات داخل المادة (الاكترونية الجزيئية والايونية) و الأشعة الكهرومغناطيسية الساقطة على المادة تؤدي إلى ظهور الخواص البصرية للمواد[4].

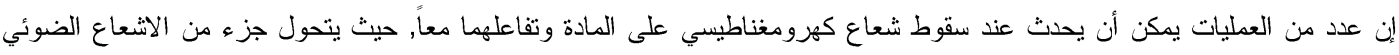

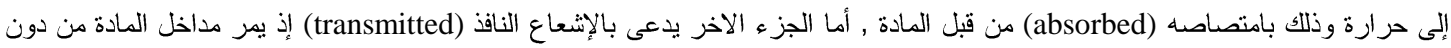
فقدان الطاقة, أما الجزء المتبقي من الإثعاع الضوئي ينعكس من سطح المادة (reflected) [5]. للحصول على معلومات عن التركيب الاخلي

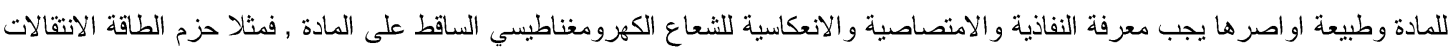
داخل المادة يتم التعرف عليها بدراسة الطيف فوق البنفسجي (Ultraviolet), أما لمعرفة مجال التطبيقات العملية التي تستخدم فيها المواد يجب البه

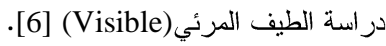

\section{Absorbance (A) الامتصاصية}

إن الكمية الرياضية التي تربط كثافة الجسيمات (التركيز ) في العينة وسمك العينة (طول المسار البصري ) هي (1)

$A=\log \left(I_{\mathrm{o}} / I\right)$.

حيث I : هي شدة الضوء عند طول الموجة ل الذي يمر خلال العينة (شدة الضوء الــنافذ) ، و Io : هي شدة الضوء قبل دخوله إلى العينة أو شدة

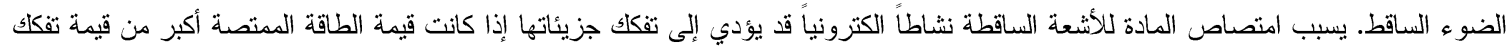

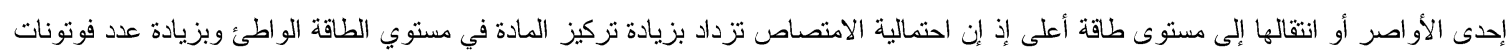

الأشعة الساقطة [6].

إن احتمالية امتصاصية الفوتون تتاسب طردياً مع تركيز الجزيئات الممتصة في العينة وسمك النموذج (طول المسار البصرب)، حسب قانون

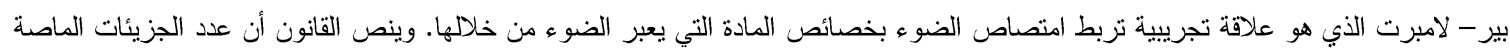

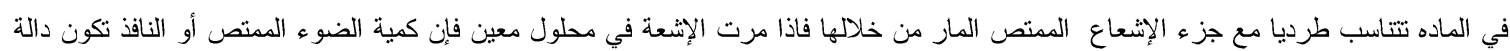
$I=I_{o} e^{-\alpha p C m \mathrm{~L}}$ أسية لتركز المذاب [7]. وكما في المعادلة التالية:

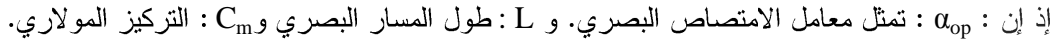
$\ln \mathrm{I}_{\mathrm{o}} / \mathrm{I}=\alpha_{\mathrm{op}} \mathrm{C}_{\mathrm{m}} \mathrm{L}=\mathrm{A}$

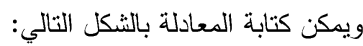
إذ يمكن تطبيق قانون بير - لامبرت Lambert - Beer Law) في مخـتلف الـــناطق الطيفية كالأشعة الفوق البنفسجية والمرئية وغيرها

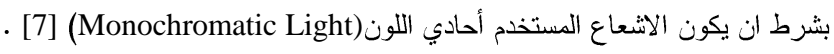

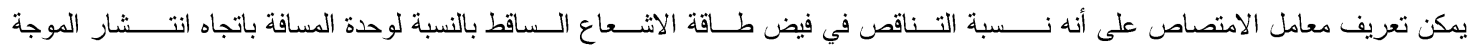

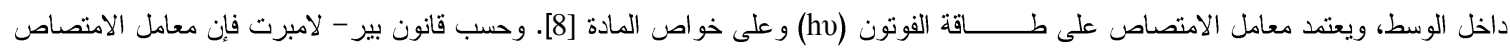

$\log \left(\mathrm{I}_{\mathrm{o}} / \mathrm{I}\right)=2.303 \mathrm{~A}=\alpha_{\mathrm{o}} \mathrm{d}$ 
تعرف النفاذية للوسط (transmittance -T) على أنها" النسبة المئوية لثدة الضوء النافذ (I) إلى شدة الضوء الساقط (I0)، أو أنها"طاقة

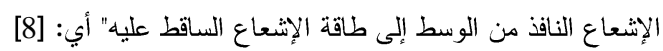

$\mathrm{T}=\left(\mathrm{I} / \mathrm{I}_{\mathrm{o}}\right)$

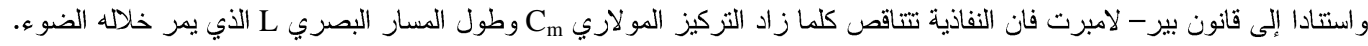
[8]: بالعلاقة الآتية

$A=-\log (1 / T)=-\log \left(I / I_{0}\right)=\log \left(I_{o} / I\right)$

و من هذه العلاقة نلاحظ إن النفاذية T تزداد كلما قلت امتصاصية A الوسط .

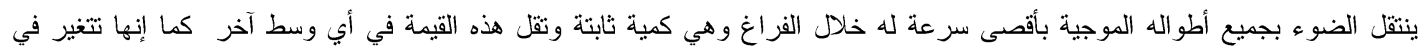
الاوساط المادية باختلاف الاطوال الموجية. وتعرف النسبة بين سرعة الضوء في الفراغ الغوال إلى سرعته في أي وسط معين لطول موجة معينه $\mathrm{n}=\mathrm{c} / \mathrm{v}$

[9]. بمعامل الانكسار الوسط لنلإك الموجة الاطوان حيث c: سرعة الضوء في الفراغ و v: سرعة الضوء في الاوساط المادية.

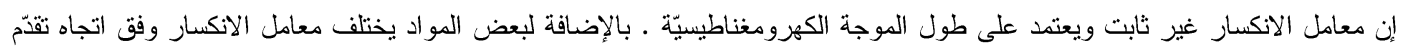

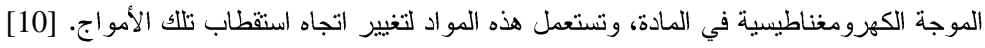

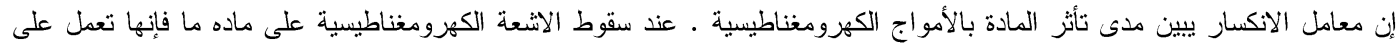

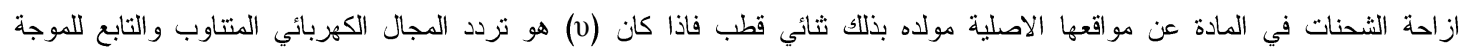
الكهرومغناطيسية الساقطة فأن الاستقطاب الكهربائي للجزيئة سوف ينذبذب بنفس التردد (v), و إن جزء من طاقة الموجة الساقطة سوف يتحول

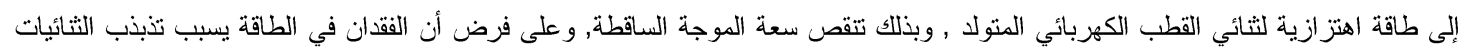

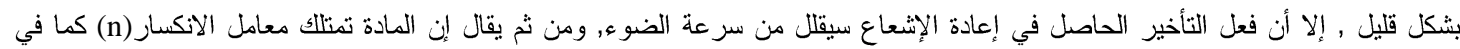

المعادلة (9).

ومن هنا يتضح إن الاستقطاب في المادة بفعل سقوط الاثعة الكهرومغناطيسية عليها مقياس لمعامل الانكسار لهذه المادة, فكلما كان

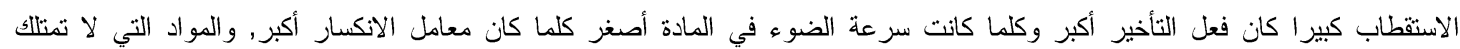
استقطاب لا تمنلك أي تأخير في إعادة الضوء, وبالتالي فإن معامل انكسارها(n=1)، وفي أغلب الحالات يكون معامل الانكسار أكبر من الواحد

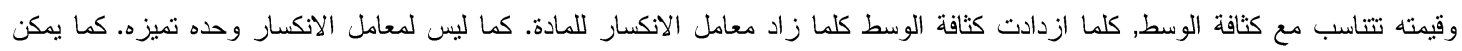
$n_{0}=\frac{1}{T}+\left[\left(\frac{1}{T^{2}}-1\right)\right]^{1 / 2}$ الحصول على معامل الانكسار من خلال المعادلة الاتية:

اما معامل الامتصاص الخطي فنجده بتطبيق المعادلة الاتية. $\alpha_{0=\frac{1}{t} \ln \left(\frac{1}{T}\right)}$ (10)

$$
\text { حيث (T) النفوذية البصرية . }
$$

\section{Spectral properties الخصائص الطيفية r. r}

ان الانتقالات الاثتعاعية هي العمليات التي ير افقها انبعاث فوتون بسبب انتقال الجزيئة المنهيجة من المستوي المتهيج إلى أحدى مستويات

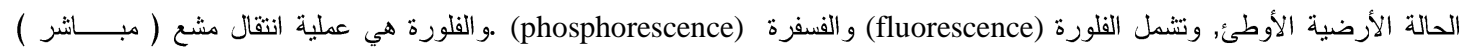

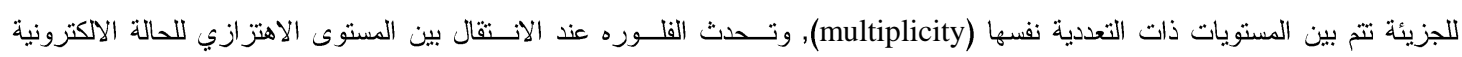

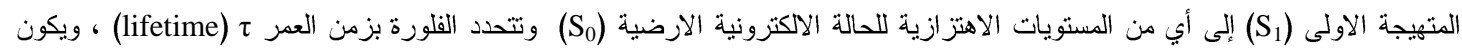
بحدود (10-9 ثانية لجزيئات الصبغة العضوية [12] ـ إلا أن بعض المركبات العضوية يحدث فيها انبعاث الفلورة من أوطأ مستوى للحالة الاكترونية المتهيجة الثانية (S2) إلى أي من المستويات الاهنز ازية للحالة الأرضية

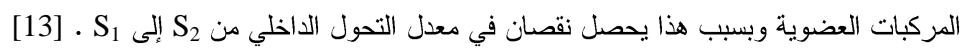


إن التركيب الجزيئي للصبغات العضوية لله أهمية في كفاءة الفلورة حيث تزداد في الجزيئات ذات التزكيب الصلد (rigid Structure)

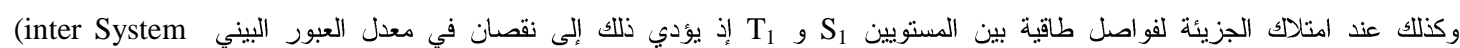
. Crossing)

وإن طيف الفلورة يحدث بسبب امتصاص الجزيئة للفوتون الساقط عليها وانتقالها إلى المستويات الآكترونية المتهيجة العليا لكن هذه

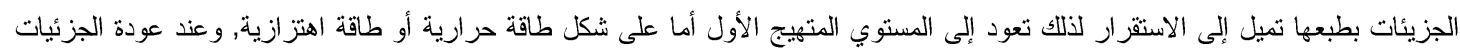

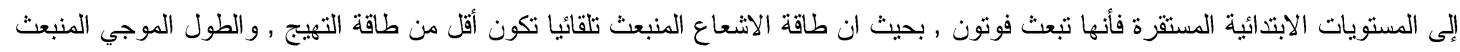
[14] [أطول من الطول الموجي المتهيج

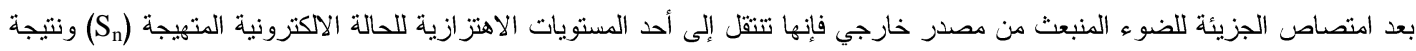

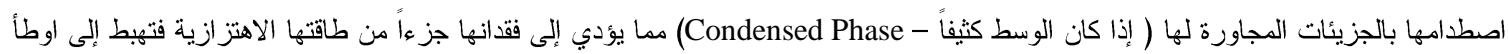

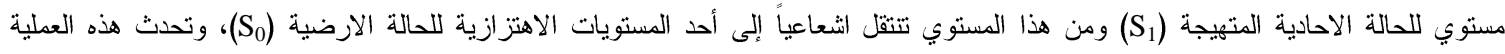

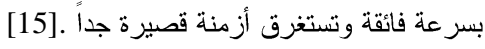

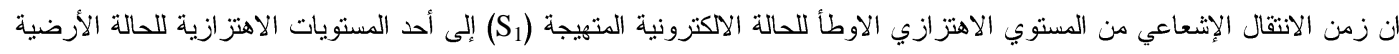

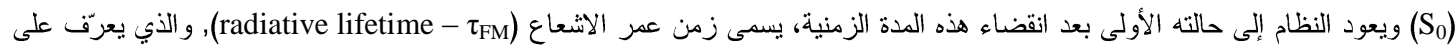

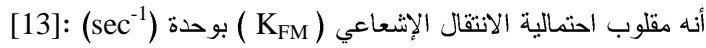

$$
\tau_{F M}=\frac{1}{K_{F M}}
$$

وبسبب وجود العمليات غير الإشعاعية المتتافسة مع احتمالية الانتقال الإثعاعي (K) فإنها سوف تقلل عدد الجزيئات المــــؤهلة لانــبعاث

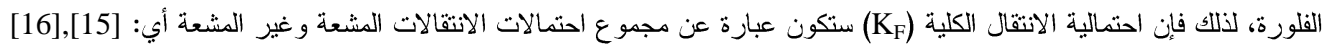

$$
\tau_{F}=\frac{1}{K_{F M}+\sum K_{d}}=\frac{1}{K_{F}}
$$

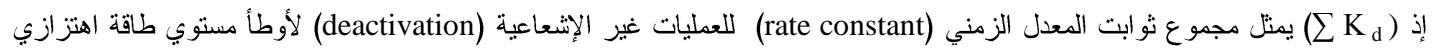

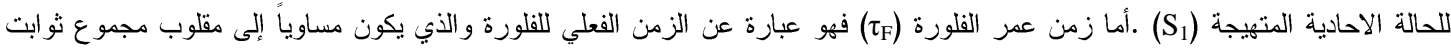

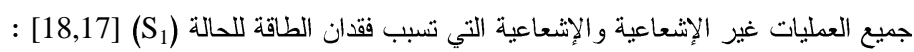
$\tau_{F}=\frac{1}{K_{F M}+K_{I C}+K_{I S C}}$

$$
\begin{aligned}
& \text { يمثل ثابت معدل التحول الداخلي. (KC) } \\
& \text { يمثل ثابت معدل العبور البيني. (KISC) }
\end{aligned}
$$

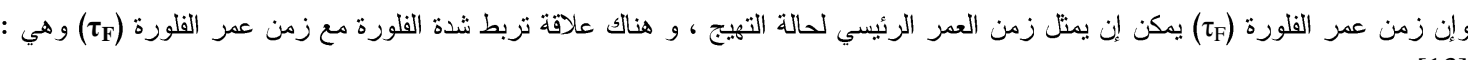

$$
I=I_{0} \exp \left(-t / \tau_{F}\right)
$$

إذ (I) تمثل شدة الفلورة عند الزمن (t)، و (t) الزمن بمثل أعلى شدة تفلور و توقف التهيج مباتثرةً .

تم تسجيل طيف الامتصاص باستخدام مطياف ثنائي الحزمة (UV-Visible - Spectrophotometer)، ويغطي هذا المطياف منطقة واسعة من الطيف الكهرومغناطيسي من المنطقة فوق البنفجية حتى منطقة تحت الحمراء القريبة، ويشتمل الجهاز على مصدرين للتهييج هما

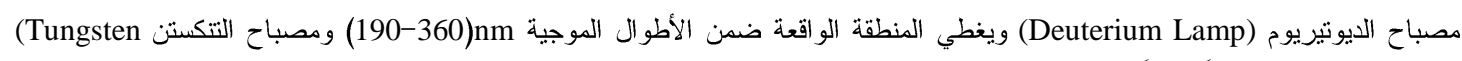

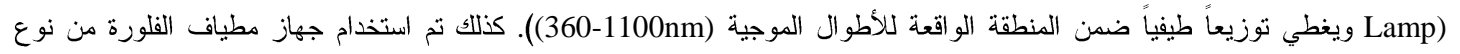

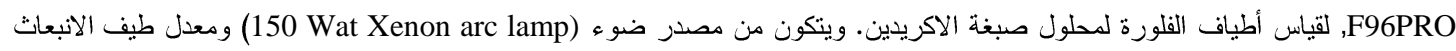

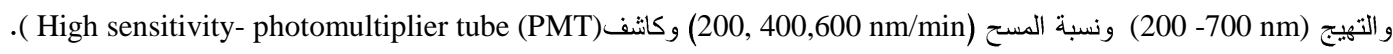




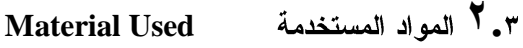

Acridine dye أ- صبغة الأكريدين

جدول(1) يبين التركيب الكيميائي للاكريدين

\begin{tabular}{|l|c|c|}
\hline Chemical Name & Molecular Formula & Molecular Weight \\
\hline Acridine Orange & $\mathrm{C}_{17} \mathrm{H}_{19} \mathrm{~N}_{3}$ & $\mathbf{2 6 5 . 3 6} \mathrm{g} / \mathrm{mol}$ \\
\hline
\end{tabular}

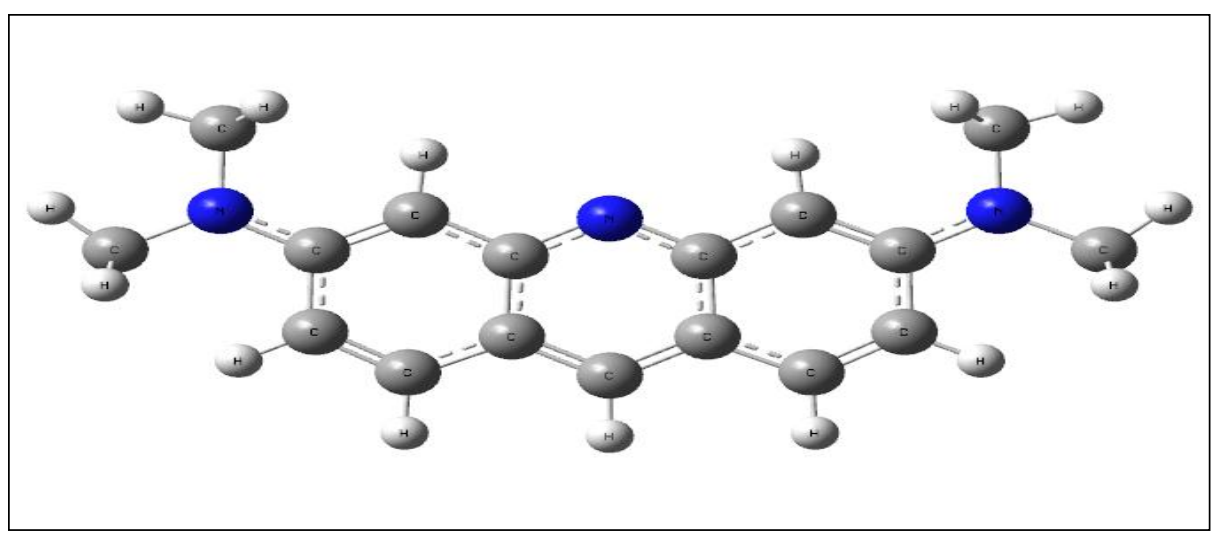

الثكل (1) التركيب الجزيئي لصبغة الاكريدين

ب - بائانول (Ethanol)

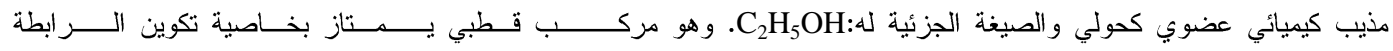

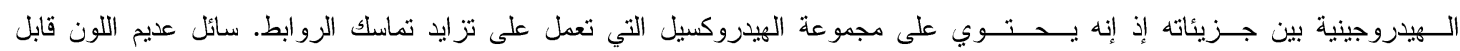

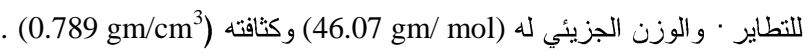

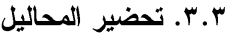

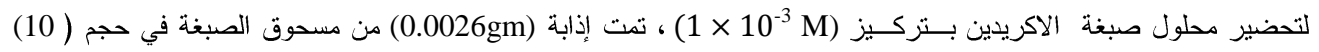

$W m=\frac{C \times V \times M \cdot W}{1000}$

cthanol) من مذيب الايثانول الذي تم استخدامه في البحث وفقاً للعلاقة:

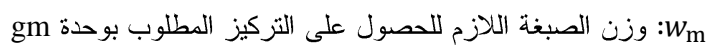

$$
\begin{aligned}
& \text { M التركيز المراد تحضيره بوحدة: C } \\
& \text { : حجم المذيب Cm }
\end{aligned}
$$

gm / mol الوزن الجزيئي للصبغة المستخدم: M.W :

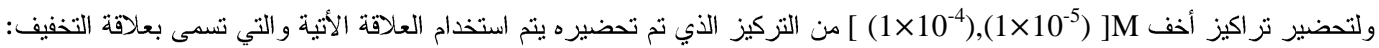
$\mathrm{C}_{1} \mathrm{~V}_{1}=\mathrm{C}_{2} \mathrm{~V}_{2}$ 


\section{Calculation and Results الحسابات و النتائج}

أظهرت نتائج أطياف الامتصاص لمحلول صبغة الاكريدين المذابة في مذيب الايثانول ولتر اكيز مختلفة

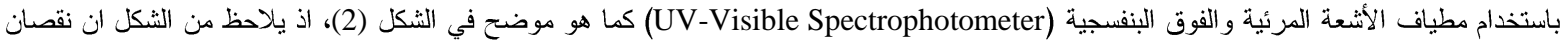

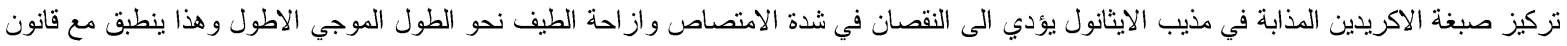
بير لامبرت. والجدول (2) يوضح تأثير التزكيز على مقادير الثندة النسبية والطول الموجي لأطياف الامتصاص لصبغة الاكريدين المذابة في الايثانول.

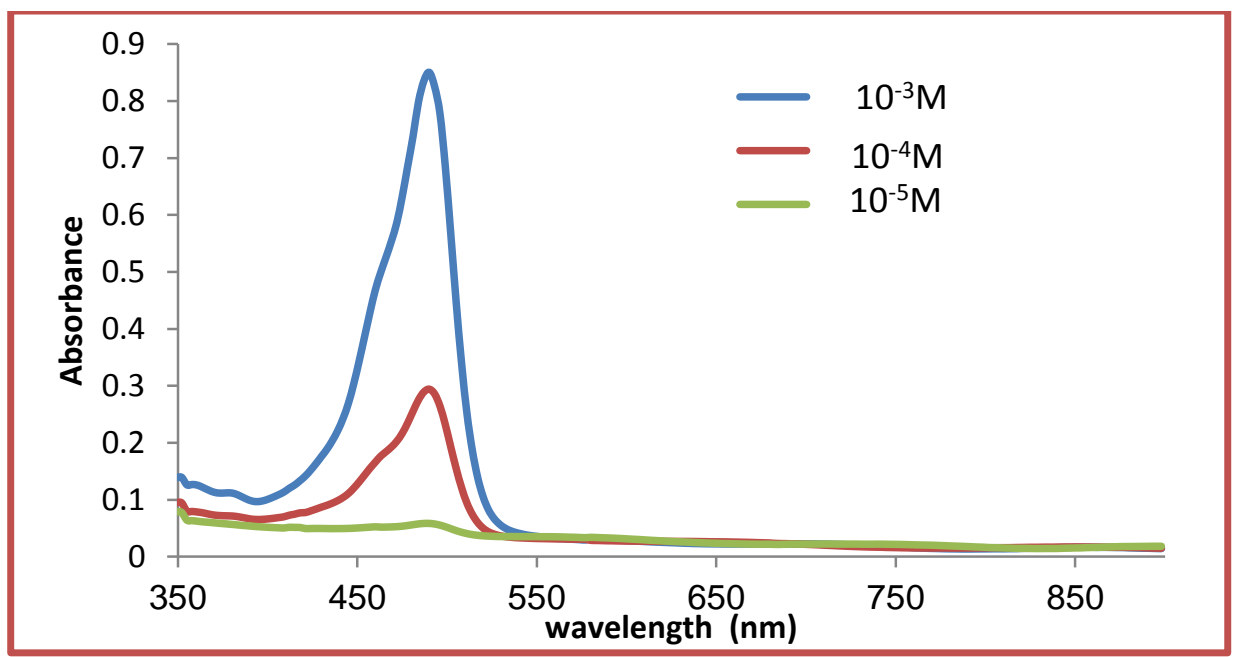

الثكل (2) طيف الامتصاص في منطقة (UV-Vis) لمحلول صبغة الاكريدين المذابة في الايثانول بتر اكيز مختلفة

جدول (2) الامتصاصية عند الأطوال الموجية العظمى لمحلول صبغة الاكريدين المذابة في الايثانول وبتر اكيز مختلفة

\begin{tabular}{|c|c|c||}
\hline $\mathrm{C}(\mathrm{M})$ & $\lambda_{\max }(\mathrm{nm})$ & $\mathrm{A}$ \\
\hline $1 \times 10^{-3}$ & 487 & $\mathbf{0 . 8 3 5 0}$ \\
\hline $1 \times 10^{-4}$ & 484 & 0.2702 \\
\hline $1 \times 10^{-5}$ & 481 & 0.0562 \\
\hline
\end{tabular}

ومن نتائج أطياف الامتصاص أمكن الحصول على أطياف النفاذية (Transmission) وكما هو موضح بالثكل (3) والجدول (3).

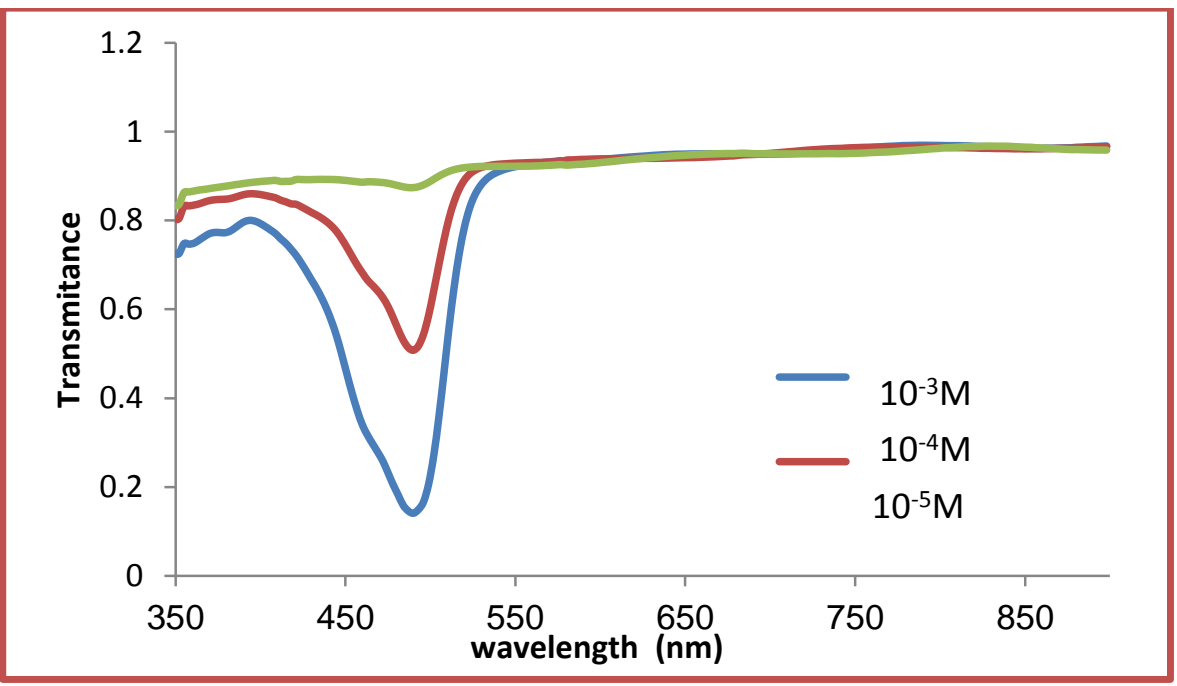

الثكل (3) طيف النفاذية في منطقة UV-Vis) لمحلول صبغة الاكريدين المذابة في الايثانول بتر اكيز مختلفة 
جدول (3) النفاذية عند الأطوال الموجية العظمى لمحلول لصبغة الاكريدين المذابة في الايثانول وبتراكيز مختلفة

\begin{tabular}{|c|c|c|}
\hline $\mathrm{C}(\mathrm{M})$ & $\lambda_{\max }(\mathrm{nm})$ & $\mathrm{T}$ \\
\hline \hline $1 \times 10^{-3}$ & 484 & 0.1591 \\
\hline \hline $1 \times 10^{-4}$ & 484 & 0.5306 \\
\hline $1 \times 10^{-5}$ & 487 & 0.8758 \\
\hline
\end{tabular}

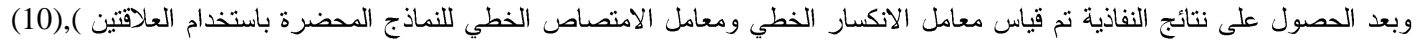

(9) فنلاحظ نقصان التركيز يؤدي إلى النقصان في معامل الانكسار ومعامل الامتصاص الخطيين كما موضح في الثكل(4) والجدول (4).

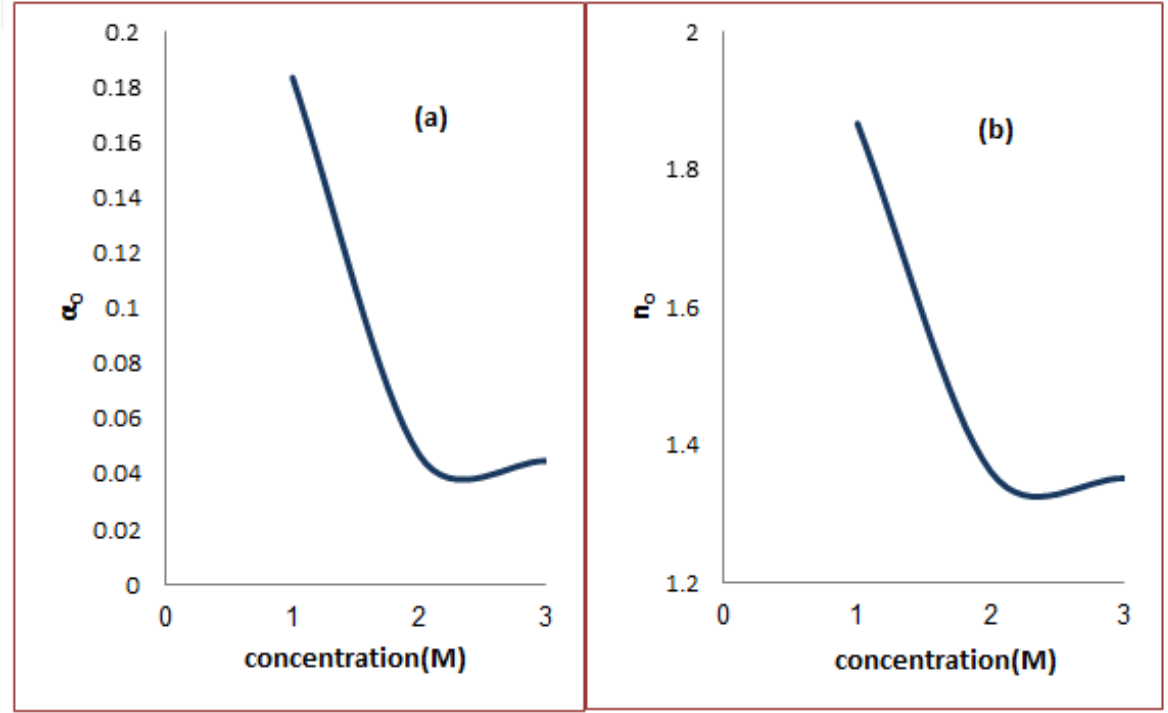

الثكل (4) (a) معامل الامتصاص الخطي (م) لمحلول صبغة الاكريلين المذابة في الايثاتول وبتراكيز المختلفة

(b) معامل الاككساز الخطي (n) لمحلول صبغة الاكريلين المذابة في الايثاتول وبتراكيز المختلفة .

جدول (4) معامل الاككسار الخطي والامتصاص الخطي مع الثر اكيز المختلفة من محلول صبغة الاكريدين المذابة في الإيثانول

\begin{tabular}{||c|c||c|c||c||}
\hline $\mathrm{C}(\mathrm{M})$ & $\begin{array}{c}\text { Wavelength } \\
(\mathrm{nm})\end{array}$ & $\begin{array}{c}\text { Linear } \\
\text { transmission }\end{array}$ & $\begin{array}{c}\text { Linear } \\
\text { Absorption } \\
\left(\alpha_{0}\right)\end{array}$ & $\begin{array}{c}\text { Linear refractive } \\
\text { index }\left(\mathbf{n}_{\mathbf{0}}\right)\end{array}$ \\
\hline \hline $1 \times 10^{-3}$ & 538 & 0.8323 & 0.18356 & 1.86750 \\
\hline \hline $1 \times 10^{-4}$ & 538 & 0.9541 & 0.04698 & 1.36200 \\
\hline \hline $1 \times 10^{-5}$ & 538 & 0.9562 & 0.04478 & 1.35192 \\
\hline
\end{tabular}

نم قياس أطياف الفلورة للنماذج المحضرة للحلول صبغة الاكريدين المذابة بالإيثانول باستخدام مطياف الفلورة (spectrofluorometer) وكانت

نــتائج القياسات كما موضح في الثنكل (5) و الجدول (5). 


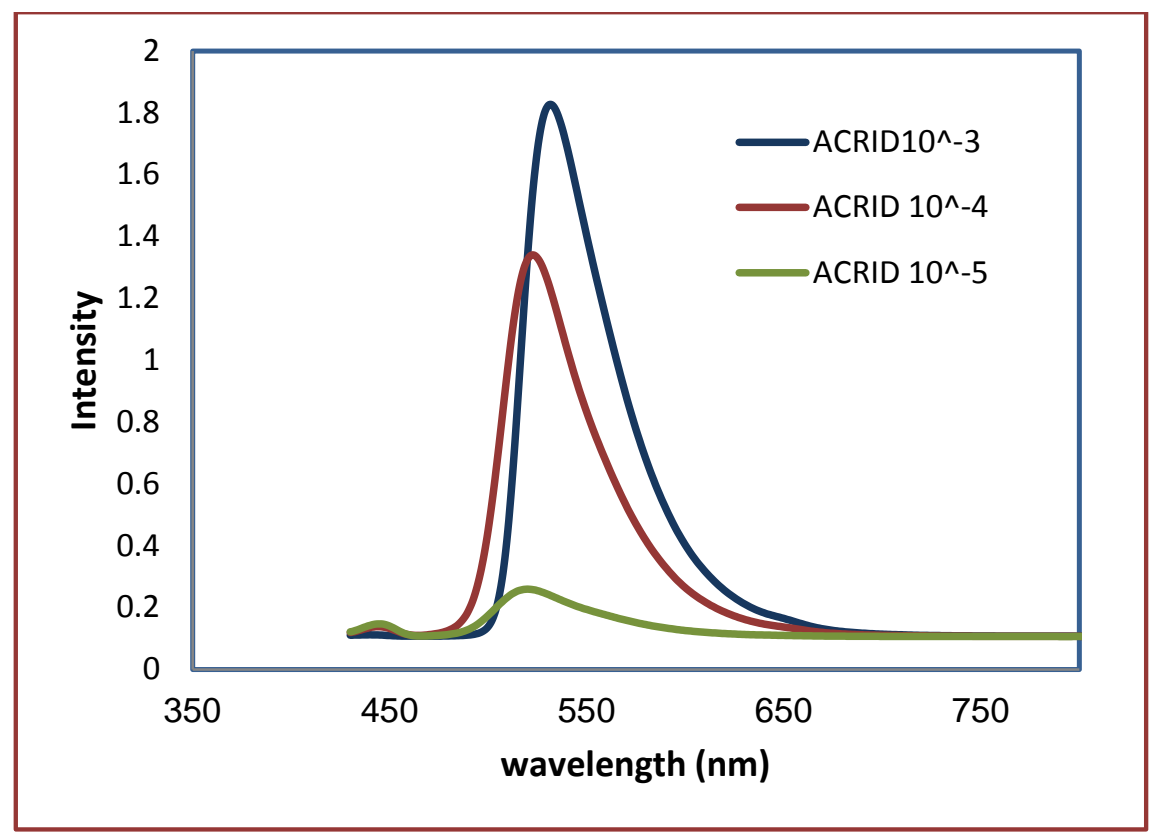

الثكل (5) منحنيات طيف الفلورة لمحلول صبغة الاكريدين المذابة في الايثانول و بتراكـيز مختلفة

جدول (5) الثدة عند الأطوال الموجية العظمى لمحلول صبغة الاكريدين المذابة في الايثانول.

\begin{tabular}{|c||c|c||}
\hline $\mathrm{C}(\mathrm{M})$ & $\lambda_{\max }(\mathrm{nm})$ & Relative Intensity \\
\hline \hline $1 \times 10^{-3}$ & 528 & 1.786 \\
\hline $1 \times 10^{-4}$ & 520 & 1.336 \\
\hline $1 \times 10^{-5}$ & 513 & 0.247 \\
\hline
\end{tabular}

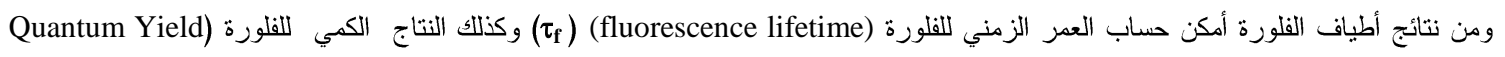

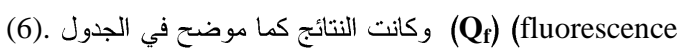

جدول (6) العمر الزمني والنتاج الكمي للفلورة وبتر اكيز مختلفة لمحلول صبغة الاكريدين

\begin{tabular}{|c||c||c|}
\hline \hline $\mathrm{C}(\mathrm{M})$ & $\tau_{\mathbf{f}}(\mathrm{ns})$ & $\mathbf{Q}_{\mathbf{f}}$ \\
\hline \hline $1 \times 10^{-3}$ & 0.287 & 0.89 \\
\hline \hline $1 \times 10^{-4}$ & 0.313 & 0.97 \\
\hline \hline $1 \times 10^{-5}$ & 0.316 & 0.98 \\
\hline
\end{tabular}

0 - 1.الاستنتاجات

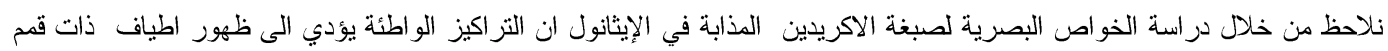

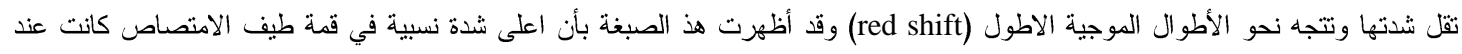

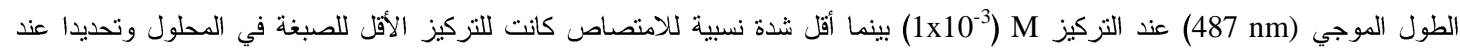

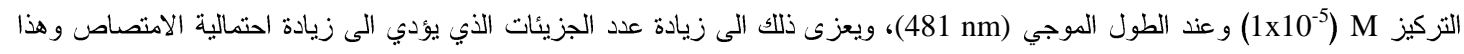

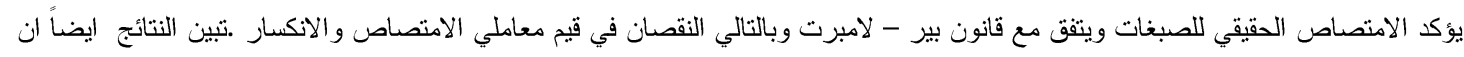

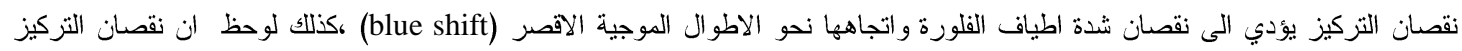


يودي الى تقليل زمن عمر الفلورة مما يؤدي ذلك الى زيادة النتاج الكمي للفلورة وبالتالي يمكن استخدام هذه الصبغة كوسط فعال في ليزرات

\section{CONFLICT OF INTERESTS}

\section{There are no conflicts of interest.}

[1] B. Snavely et al. ," Organic Molecular Photo physics", Vol.1, John Wiley \& Sons , p. 239, (1973).

[2] F. Duarte ,"Tunable Laser Optics" Elsevier Academic, New York, Appendix of Laser Dyes ,(2003).

[3] J. Mc Murry ," Organic Chemistry", Oxford university Press, New York ,p. 314, (1984).

$$
\text { [4] حسن الثربيني, بتول الخياط , صبحي كمال حسون, " البصريات الفيزيائية"، جامعة بغداد - كلية التربية (r (1)). }
$$

[5] R. Fowels, " Introduction to modern optics", holt Rinehart and Winston ,Inc., second , (1975) .

[6] M. Wang, Z. Lin and G. Zhai, "Optical linearity and nonlinearity of ZnSe Nano crystals embedded in epoxy resin matrix investigated by z-Scan technique" , Ceramics International , 34, 1073-1076,(2008).

[7] Verhaegen et al. , "Mass spectrometric determination energy of $B_{2}$ ", J. Chem. Phys. ,37,1367, (1960).

[8] J.M. ,"Modern Spectroscopy" John Wiley and sons Ltd, The Atrium, Southern Cate Chionester, west Sussex PO19 8SQ, England ,(2004).

[9] M. Parikh, "Absorption Spectroscopy of Organic Molecules", Adison-Weleg, 1974 .

[10] W. Demtröder, "Atoms ,Molecules and Photons", Springer Berlin Heidelberg New York. 2010.

[11] B. Straughan et al. , "Spectroscopy" , Chapman and Hall, London,(1976).

[12] S. Jacobs, "Laser Application to Optics and Spectroscopy", Addison-Wesly Publishing Company, London. Amsterdam .Don Mills, Ontario-Sydney-Tokyo, $2^{\text {nd }}$ edition, (1975).

[13] M. Maeda, "Laser Dyes, Properties of organic compounds for dye lasers “, Academic Press, INC, (1984).

[14] R. Sastre," Laser performance of Coumarin 540A dye molecules in polymeric host media with different viscosities: From liquid solution to solid polymer matrix ", J. Appl. Phys., Vol.83, No.2, pp.650-660, (1998) .

[15] N. Sahib ," Spectroscopic Study for Some Organic Dyes " , A Thesis , Al-Mustansiriya University, College of Sciences(2005).

[16] R. Hamood Al-Hamadani ," Spectroscopy Study and Manfacturing of An Active Polymeric Laser Medium " , A Thesis ,University of Baghdad, College of Science for Women, (2006).

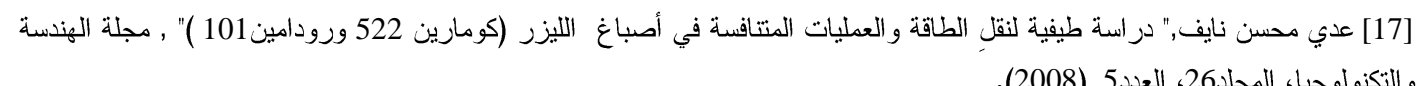$$
\text { والتكنولوجيا، المجلد26، العدد5 , (2008). }
$$

[18] رافد عباس علي," قياس أطياف الامتصاص و الفلورة لصبغة الكومارين في مذيب التلوين", مجلة جامعة ذي قار , العدد1, المجلد5), (2009). 